Published in final edited form as:

Transfusion. 2013 March ; 53(3): 539-544. doi:10.1111/j.1537-2995.2012.03754.x.

\title{
The low prevalence $\mathrm{Rh}$ antigen STEM (RH49) is encoded by two different RHCE*ce818T alleles that are often in cis to $R H D^{\star} D O L$
}

\author{
Marion E. Reid ${ }^{1,2}$, Christine Halter Hipsky ${ }^{1}$, Kim Hue-Roye $^{1}$, Gail Coghlan ${ }^{3}$, Coral Olsen ${ }^{4}$, \\ and Christine Lomas-Francis ${ }^{2}$ \\ ${ }^{1}$ Laboratory of Immunochemistry, New York Blood Center, New York, NY \\ 2Laboratory of Immunohematology and Genomics, New York Blood Center, Long Island City, NY \\ ${ }^{3} \mathrm{Rh}$ Laboratory, Department of Pediatrics and Child Health, University of Manitoba, Winnipeg, \\ Manitoba, Canada \\ ${ }^{4}$ Specialized Laboratory Services, South African National Blood Service, Pinetown, KwaZulu- \\ Natal, South Africa
}

\begin{abstract}
Background-STEM (RH49) is a low prevalence antigen in the Rh blood group system. A scarcity of anti-STEM has precluded extensive study of this antigen. We report that two alleles with a $R H C E^{*} c e 818 C>T$ change encode a partial e, and a $\mathrm{hr}^{\mathrm{S}}-, \mathrm{hr}^{\mathrm{B}}+, \mathrm{STEM}+$ phenotype and that both alleles are frequently in cis to $R H D^{*} D O L 1$ or $R H D^{*} D O L 2$.
\end{abstract}

Materials and methods-Blood samples were from donors and patients in our collections. Hemagglutination, DNA and RNA testing was performed by standard techniques.

Results-Fourteen STEM+ samples were heterozygous $R H C E^{*} c e 818 C / T$ : six had $R H C E^{*} c e B I$ and eight had a novel allele, $R H C E^{*} c e S M$. Eleven were heterozygous for $R H D^{*} D O L 1$ or $R H D^{*} D O L 2$. Eleven samples, previously typed STEM-, had $R H C E^{*} c e 818 C / C$ (consensus nucleotide). RBCs from informative STEM+ samples were $\mathrm{e}+/-\mathrm{hr}^{\mathrm{S}}-\mathrm{hr}^{\mathrm{B}}+$. One person who was heterozygous $R H C E^{*} c e B I$ and $R H C E^{*} c E$ had an anti-e-like antibody in her plasma, and one person, who was hemizygous for $R H D^{*} D O L 2$ had anti-D in her plasma.

Conclusions-We show that two alleles with a $R H C E^{*} c e 818 C>T$ change ( $R H C E^{*} c e B I$ and $R H C E^{*}(e S M)$ encode a $\mathrm{hr}^{\mathrm{S}}-\mathrm{hr}^{\mathrm{B}}+\mathrm{STEM}+$ phenotype. In addition, both alleles are frequently in cis to $R H D^{*} D O L 1$ or $R H D^{*} D O L 2$ and $R H C E^{*} c e B I$ encodes a partial e antigen. In the small cohort of samples tested, $R H D^{*} D O L$ invariably traveled with $R H C E^{*} c e 818 T$. Our study also confirmed the presumption that $R H D * D O L 2$, like $R H D * D O L 1$, encodes a partial D antigen and the low prevalence antigen DAK.

\section{Keywords}

Rh blood group system; RHCE variant; partial D phenotype; low prevalence Rh antigen; STEM

Corresponding author: Marion E Reid, PhD Laboratory of Immunochemistry, New York Blood Center 310 East $67^{\text {th }}$ Street, New York, NY 10065 212-570-3294 / Fax: 212-737-4935 mreid@nybloodcenter.org.

The authors certify that they have no affiliation with or financial involvement in any organization or entity with a direct financial interest in the subject matter or materials discussed in this paper. 


\section{Introduction}

STEM (RH49) is a serologically defined low prevalence antigen that was shown, by family studies, to be in the Rh blood group system. ${ }^{1}$ A scarcity of monospecific anti-STEM has precluded extensive study of this antigen. An article by Marais and coworkers published in $1993^{1}$ is the sole original report in the literature regarding STEM and anti-STEM. These authors reported that STEM has a variable expression, which is an inherited characteristic, and is associated with an altered e antigen. They also found that RBCs from approximately $65 \%$ of $\mathrm{hr}^{\mathrm{S}}$ - and $30 \%$ of $\mathrm{hr}^{\mathrm{B}}$ - South African donors typed STEM+ and that anti-STEM induced mild hemolytic disease of the fetus and newborn (HDFN). ${ }^{1}$

$R H D$ and $R H C E$ are well studied and numerous variants, and the phenotypes they encode, have been described. ${ }^{2-4}$ The high degree of diversity is a consequence of the homology between RHD and RHCE, their opposite orientation and close proximity on chromosome $1 \mathrm{p}$, which favor the exchange of nucleotides. Some of the variant alleles encode so-called partial antigens, which are identified when an antigen-positive person makes the corresponding alloantibody. Several variant RHD and RHCE alleles have been shown, especially in African Americans, to be inherited en bloc. Examples, represented by the transfusion medicine-friendly allele names that have been proposed by the ISBT Working Party on Red Cell Immunogenetics and Blood Group Terminology (see ISBT-web.org), are $R H D * D A R-R H C E^{*} c e A R, R H D^{*} D I I I a-R H C E^{*} c e S$, and $R H D^{*} D A U-R H C E^{*} c e M O{ }^{5}$ Variant phenotypes can express more than one low prevalence antigen, e.g., $R H D^{*} D I I I a-$ $R H C E * c e S$ encodes V, VS, and DAK. Similarly, a low prevalence antigen can be encoded by more than one allele, e.g., DAK is expressed on RBCs with the DIIIa, $\mathrm{R}^{\mathrm{N}}$, or DOL phenotypes that are encoded by $R H D^{*}$ DIIIa, $R H C E^{*} C e R N, R H D * D O L 1$, and $R H D^{*} D O L 2$, respectively. ${ }^{6}$

We report here an expansion of our initial findings ${ }^{7-9}$ that two alleles with a $R H C E^{*} c e 818 C>T$ change encode the e+/- $\mathrm{hr}^{\mathrm{S}}-\mathrm{STEM}+$ phenotype. These alleles are (i) the previously reported $R H C E^{*} c e B I^{10}$ (IBST provisional name $R H C E^{*} 01.08$, or $R H C E^{*} c e .08$ ), which was previously not known to encode a novel low prevalence antigen, and (ii) the novel allele, $R H C E^{*} c e S M$, (ISBT provisional name $R H C E^{*} 01.09$, or $R H C E^{*} c e .09$ ). Both alleles are frequently in cis to $R H D^{*} D O L 1$ (ISBT provisional name $R H D^{*} 12.01$ ) or $R H D^{*} D O L 2$ (ISBT provisional name $R H D^{*} 12.02$ ). Furthermore, we confirm that $R H C E^{*} c e B I$ encodes a partial e antigen, and that, like $R H D^{*} D O L 1, R H D * D O L 2$ encodes a partial $\mathrm{D}$ antigen and the DAK antigen.

\section{Material and Methods}

\section{Samples and hemagglutination testing}

Blood samples were freshly collected samples or were recovered from storage in liquid nitrogen. They were either referred for investigation, found in DNA screening tests, or received from numerous colleagues over many years. Reagent RBCs and antibodies were from our collections. Hemagglutination was performed by methods that were optimal for the antibody being used. STEM typings were determined in our laboratories.

\section{Analyses of DNA and RNA}

RNA was isolated from the reticulocytes by standard methods using TriZol and PureLink RNA Mini Kit (Invitrogen, Carlsbad, CA). Reverse transcription was carried out with genespecific $R H D$ and $R H C E$ primers and Superscript III according to manufacturer's instructions (Superscript III first-strand synthesis SuperMix, Invitrogen), and polymerase chain reaction (PCR) amplification was carried out for 35 cycles with primers to amplify exons 1 to 4 and exons 5 to 10 in $R H D$ and $R H C E$ as described previously. ${ }^{11}$ RT-PCR 
products were checked for appropriate size and purity on agarose gels, purified using PCR product clean-up reagent according to manufacturer's instructions (ExoSAP-IT, USB Corporation, Cleveland, OH), and directly sequenced by GENEWIZ, Inc. (South Plainfield, $\mathrm{NJ})$. Sequences were aligned, and protein sequence comparisons were performed with Sequencher v4.9 (GeneCodes, Ann Arbor, MI).

For those blood samples where RNA extraction failed, and for confirmatory testing, genomic DNA was isolated using the QIAamp DNA Blood Mini Kit (QIAGEN, Inc. Valenica, CA). Samples were tested for $R H C E^{*} c e B I$ and $R H C E^{*} c e S M$ by analyzing $R H C E^{*} c e 818 C / T$ and $R H C E^{*} c e 1132 C / G$ on PCR amplicons using $R H C E$ exon-specific primers as previously described, ${ }^{12}$ followed by sequencing by GENEWIZ, Inc. To screen for $R H D^{*} D O L 1$, and $R H D^{*} D O L 2$, genomic DNA was amplified using specific primers flanking $R H D$ exon 4 and exon 8 as previously described, ${ }^{12}$ and products were submitted for sequencing to GENEWIZ, Inc to determine the presence of $R H D^{*} D O L 1$ (nt 509T $>\mathrm{C}$ in exon 4) and $R H D^{*} D O L 2$ (nt 509T $>\mathrm{C}$ in exon 4 and nt $1132 \mathrm{C}>\mathrm{G}$ in exon 8 ). The $R H D^{*} 410 T>C$ nucleotide change in exon 3 that would discriminate $R H D * D O L 3$, was not analyzed.

$R H D$ zygosity analyses were performed to detect the presence or absence of the hybrid box as previously reported. ${ }^{13}$

\section{Results}

\section{Two alleles encode STEM and are linked to RHD*DOL}

$R H D$ and $R H C E$ cDNA, prepared from samples with a historic STEM+ or STEM- type, were sequenced. We found that two RHCE alleles (RHCE*ceBI or RHCE*ceSM) and two RHD alleles $\left(R H D^{*} D O L 1\right.$ or $\left.R H D^{*} D O L 2\right)$ were present in the STEM+ samples. We then tested samples from people of African origin for the presence of these alleles using a combination of PCR-based assays (see Material and methods section).

Taken together, a total of fourteen STEM+ samples were tested: all were heterozygous for $R H C E^{*} c e 818 C / T$ : six (including the original index case, Stemper) were $R H C E^{*} c e B I$ [ce48C (16Cys), 712G (238Val), 818T (273Val), 1132G (378Val)] ${ }^{10}$ and eight had a new allele, which we name $R H C E^{*} c e S M$ [ce 48C (16Cys), 712G (238Val), 818T (273Val); so named from the first and last letters of 'STEM']. Although $R H C E^{*} c e B I$ has been reported ${ }^{10}$, it previously had not been shown to encode the STEM antigen. Of the six samples with $R H C E^{*} c e B I$, three were heterozygous for $R H D^{*} D O L 1$ [509C (170Thr), 667G (223Val)] and three were heterozygous for $R H D^{*} D O L 2$ [509C (170Thr), 667G (223Val), $1132 \mathrm{G}$ (378Val)]. Of the eight samples with $R H C E * c e S M$, five were heterozygous for $R H D^{*} D O L 1$, and three did not have either a RHD*DOL1 or a RHD*DOL2 allele (see Table $1)$.

Four additional samples, that could not be tested with anti-STEM (because the RBCs did not recover from cryopreservation and fresh samples were not available), were heterozygous for $R H C E^{*} c e B I$ and heterozygous for $R H D^{*} D O L 1$ (Table 1). Thus, of the 18 samples with a $R H C E^{*} c e 818 C>T$ change, 12 had $R H D^{*} D O L 1$, three (including the original index case) had $R H D^{*} D O L 2$, and three did not have a RHD*DOL allele (Tables 1 and 2).

Two samples from two South African donors (code names, Roman and Alson) were historically typed as STEM $+^{\mathrm{w}}$. However, these samples did not have $R H C E^{*} c e 818 T$ or $R H D * 509 C, 667 G$. The reason for this apparent discrepancy could not be determined because our concerted efforts to obtain fresh samples for extensive testing have been unsuccessful and other samples with this unique genotype were not available. Both RBC 
samples were non reactive by the direct antiglobulin test. As the STEM typing of both samples was considerably weaker than that of samples with $R H C E^{*} c e 818 T$, it is highly probable that the agglutination was caused by an additional, but unidentified, antibody in the test plasma and was not due to the anti-STEM. A paucity of plasma containing anti-STEM precluded us from being able to perform absorption and elution studies.

As few samples tested for STEM, either positive or negative, are available, we have included our testing of eleven STEM- samples, which included $\mathrm{hr}^{\mathrm{S}}-$ and $\mathrm{hr}^{\mathrm{B}}$ - samples; all had the consensus nucleotide, i.e., $R H C E^{*} c e 818 C / C$. The RHD and RHCE alleles associated with these STEM- samples are given in Table 1. Attempts to perform $R H D$ zygosity testing confirmed that discrepant results are frequently obtained in the presence of an RHD*DOL allele and, thus the results are not given. ${ }^{14-16}$

\section{STEM+ and variant e antigen}

Hemagglutination results given in Table 1 are a compilation of those obtained from historic records and those performed during this study. Accessibility of RBCs, the condition of the RBC sample, or lack of anti-STEM prevented us from being able to test all samples.

RBCs from eight informative STEM+ samples [with a hr ${ }^{\mathrm{S}}$ - haplotype $\left(R H C E^{*} c E\right.$, $R H C E^{*} c e J A L, R H C E^{*} c e A R, R H C E^{*} c e E K$, or $R H C E^{*} c e M O$ ) in trans to the $R H C E^{*} c e B I$ or $R H C E^{*}(e S M)$ ] were tested by hemagglutination and found to be $\mathrm{hr}^{\mathrm{S}}$-. In contrast, $\mathrm{RBCs}$ from the only two informative samples with $\mathrm{a} \mathrm{hr}^{\mathrm{B}}$ - haplotype $\left(R H C E^{*} C E\right)$ in trans to $R H C E^{*} c e B I$ were $\mathrm{hr}^{\mathrm{B}}+$. RBCs from the original index case (Stemper) and from another $R H C E^{*} c e B I / R H C E^{*} c E$ sample (MA72-09) carried a single dose of the variant e antigen, they were agglutinated by four commercial anti-e and five single clone monoclonal anti-e (MS16, MS21, MS69, HIRO41, HIRO43), but were not agglutinated by three other monoclonal anti-e (MS19, MS62, MS63), indicating that $R H C E^{*} c e 818 T$ encodes an altered e antigen. One person (MA72-09) with $R H C E^{*} c e B I / R H C E^{*} c E$ had anti-e-like antibodies in his plasma, providing the first evidence that $R H C E^{*} c e B I$ encodes a partial e antigen.

\section{RHD*DOL encodes a partial D}

One person (MA170-09) with $R H D^{*} D O L 2$ (presumed to be hemizygous) had anti-D in her plasma. Given the similarity in D epitope expression between DOL1 and DOL2, the presumption can be made that DOL2 is also a partial D but the presence of anti-D in this patient provides the first actual evidence that this allele encodes a partial D antigen. One person (MG303-00) with $R H D^{*} D O L 1$ had anti-D in her plasma, confirming that this allele encodes a partial $\mathrm{D}$ antigen.

\section{DAK typing}

All of 14 STEM+ samples tested with anti-DAK (Akom, Riz, Jep), were positive. Twelve gave strong reactions: nine had $R H D^{*} D O L 1$, two had $R H D^{*} D O L 2$, one had $R H D /$ $R H D^{*}$ DIIIa. Two gave weak reactions: both had the RHD*weak D type 4.2.2/RHD*D186T haplotype $^{17}$ (Table 1). RBCs with DOL or DIIIa phenotypes have been previously shown to express DAK. ${ }^{6}$ The DAK+ result on RBCs from two samples with $R H{ }^{*} D O L 2$ (Stemper and RFA-NYBC221) showed that this allele, like $R H D * D O L 1$, encodes DAK. The unexpected reactivity of anti-DAK, albeit weak, with RBCs from the two samples with $R H D^{*}$ weak $D$ type $4.2 .2 / R H D^{*} 186 T$ is likely due to the $186 \mathrm{G}>\mathrm{T}$ change that is predicted to encode Phe instead of Leu at amino acid residue 62, because this change is present on RBCs with the DIIIa phenotype. However, more samples need to be tested to confirm this hypothesis. The RHD*D186T allele is novel and we are reporting it elsewhere. ${ }^{17}$ 


\section{Discussion}

We have identified two RHCE alleles, both with a change of nucleotide $818 \mathrm{C}>\mathrm{T}$ in $R H C E^{*} c e$, that encode the STEM antigen. One allele $\left(R H C E^{*} c e B I\right)$ has been previously reported but was not known to be associated with STEM, ${ }^{10}$ and the other is a novel allele $\left(R H C E^{*} c e S M\right)$. Of the 18 samples with $R H C E^{*} c e 818 C / T, 12$ had $R H D^{*} D O L 1$ and three had $R H D^{*} D O L 2$ (hereafter collectively called $R H D^{*} D O L$ ). Thus, in this small cohort of 18 samples, $R H D * D O L$ travelled with one of the two $R H C E^{*} c e 818 T$ variants in 15 cases. Three samples with $R H C E^{*} c e 818 C / T$ but not $R H D^{*} D O L$ were STEM+ and provided evidence that STEM is encoded by the $R H C E^{*} c e 818 T$ variant alleles and not by $R H D^{*} D O L$. Two of these samples had both $R H D^{*}$ weak $D$ type 4.2.2 and the novel allele, $R H D * D 186 T{ }^{17}$ The third sample had $R H D / R H D * D I I I$.

In a separate, previously reported, study, we tested 705 samples that were not serologically typed for STEM and found four had $R H C E^{*} 818 C / T$. $^{9}$ Of these 705 samples, 70 samples were from patients with sickle cell disease and 220 samples were from blood donors who self-identified as being of African descent. Two of the 220 donors were heterozygous for RHCE*818C/T and 1132G (RHCE*ceBI); both samples had RHD/RHD*DOL. In these 290 samples, no other RHD*DOL were found. This shows the alleles encoding STEM are not as rare as previously thought, an impression due in part, no doubt, to the dearth of anti-STEM. Predicting the STEM type of samples by DNA analyses provides a means to have appropriate RBCs available to aid in the identification of anti-STEM, to provide matched blood products to immunized patients, and to overcome the problem of the scarcity of antiSTEM. Furthermore, testing plasma from patients who have been transfused with blood predicted to be STEM+ could reveal a source of anti-STEM and/or anti-DAK.

Our serologic tests indicate that $R H C E^{*} 818 T$ does not produce $\mathrm{hr}^{\mathrm{s}}$ and the plasma of one STEM+ person contained an anti-e-like antibody. The exact specificity of this antibody remains to be determined. Other $\mathrm{hr}^{\mathrm{S}}$ - RBCs exist, namely those with ceAR, ceMO, and ceEK phenotypes, but there is no evidence that these RBCs are STEM+. The anti-hr ${ }^{\mathrm{S}}$ made by $\mathrm{hr}^{\mathrm{S}}$ - STEM- people may not be mutually compatible and are likely to be incompatible with $\mathrm{hr}^{\mathrm{S}}-\mathrm{STEM}+\mathrm{RBCs}$, and vice versa. Indeed, Peyrard, et al. briefly reported that the antibody made by a $R H C E^{*} c e M O / R H C E^{*} c e M O$ person reacted strongly (4+) with RBCs from a $R H C E^{*} c e B I / R H C E^{*} c e B I$ person. ${ }^{18}$ Neither ceBI nor ceSM express $\mathrm{hr}^{\mathrm{S}}$ but, based on two informative samples, do express $\mathrm{hr}^{\mathrm{B}}$. Thus, it is clear why the original study ${ }^{1}$ found that approximately $65 \%$ of $\mathrm{hr}^{\mathrm{S}}$ - samples were STEM+ but it is unclear why it found that approximately $30 \% \mathrm{of} \mathrm{hr}^{\mathrm{B}}$ - samples were STEM+.

Depending on alleles carried on the in trans chromosome, patients with the $R H D^{*} D O L R H C E^{*} c e 818 T$ haplotypes can make anti-D, anti-e-like/-hr ${ }^{\mathrm{S}}$, or anti-Rh18 10 and their RBCs carry the low prevalence antigens STEM and DAK. The original JAL+ proband (J. Allen) has $R H D^{*} D / R H D * D O L 1$ and $R H C E^{*} c e J A L / R H C E^{*} c e B I$ and his RBCs express JAL, STEM, DAK, and extremely weak V and VS. ${ }^{19}$ In our relatively small study, two other samples (Delia and Keys) harbored the RHCE*ceJAL allele associated with expression of JAL. Our results highlight that many RBCs express multiple low prevalence antigens and this fact should be taken into account when identifying antibodies. From our results, most RBCs expressing STEM also express DAK and a high proportion express V and VS. It is possible that some antibodies have been misidentified and retesting them with a panel of RBC samples from people whose allele makeup has been determined will help to fine-tune the specificity(ies) within a given plasma. Having appropriate RBCs to test would resolve the conundrum of exactly which proteins express DAK. 
In summary, our study revealed several new findings: $R H C E^{*} c e B I$ and $R H C E^{*} c e S M$ encode STEM; on limited testing, $R H C E^{*} c e B I$ encodes a slightly stronger expression of STEM than does $R H C E^{*} c e S M$. This is consistent with the previously reported variable expression ${ }^{1}$ and provides evidence that STEM is encoded by these variant $R H C E$ alleles and not the $R H D^{*} D O L$. $R H C E^{*} c e B I$ and $R H C E^{*} c e S M$, which encode a partial e and lack of hr ${ }^{\mathrm{S}}$ are often, although not invariably, in cis to $R H D^{*} D O L 1$ or $R H D^{*} D O L 2$. In this study and our previously reported study ${ }^{9}$ in all samples with $R H D^{*} D O L, R H C E^{*} c e B I$ or $R H C E^{*} c e S M$ were found in cis.

The variant $\mathrm{RhD}$ encoded by $R H D^{*} D O L 2$, is a partial $\mathrm{D}$ antigen. As anti-STEM is in short supply, typing for this antigen by hemagglutination is virtually impossible; however, DNAbased assays provide a useful tool to predict the presence of STEM. Knowledge of the molecular basis of various Rh antigens should help to not only establish which antigenic determinants are encoded by which alleles but also to accurately establish the specificity of an antibody, or antibodies, in a plasma. In table 1, when possible, the donor code name is used so that others who have these samples in their frozen library can use the knowledge of which alleles are present to aid selection of appropriate RBCs for inclusion on a panel used for antibody identification purposes.

\section{Acknowledgments}

We thank Elizabeth Smart, from Specialized Laboratory Services, South African National Blood Service, for many of the samples used and numerous colleagues for providing samples, the technologists in the Laboratory of Immunohematology for serological testing, and Robert Ratner for help in preparing this manuscript. This study was supported in part by grant NIH HL091030 (CHH, MER, KHR), and the Winnipeg Rh Institute Foundation (GC).

\section{References}

1. Marais I, Moores P, Smart E, Martell R. STEM, a new low-frequency Rh antigen associated with the e- variant phenotypes $\mathrm{hr}^{\mathrm{S}}$-(Rh: - 18, -19) and $\mathrm{hr}^{\mathrm{B}}$-(Rh: -31, -34). Transf.Med. 1993; 3:35-41.

2. Reid, ME.; Lomas-Francis, C. Blood Group Antigen FactsBook. 2nd ed. Academic Press; San Diego: 2004.

3. Flegel, WA. The Rhesus site: Explore the intricacies of the most complex blood group gene locus. Institut für Klinische Transfusionsmedizin und Immungenetik Ulm; Ulm, Germany: 2007. [website]http://www.uni-ulm.de/\%7Ewflegel/RH/

4. Human Genome Variation Society. Blood Group Antigen Gene Mutation Database. 2006. [website]http://www.ncbi.nlm.nih.gov/gv/mhc/xslcgi.cgi?cmd=bgmut/home

5. Westhoff CM, Vege S, Halter-Hipsky C, Whorley T, Hue-Roye K, Lomas-Francis C, Reid ME. DIIIa and DIII Type 5 are encoded by the same allele and are associated with altered RHCE*ce alleles: clinical implications. Transfusion. 2010; 50:1303-11. [PubMed: 20088832]

6. Reid ME, Storry JR, Sausais L, Tossas E, Rios M, Hue-Roye K, Gloster ES, Miller ST, Wolf C, Lomas-Francis C. DAK, a new low-incidence antigen in the Rh blood group system. Transfusion. 2003; 43:1394-7. [PubMed: 14507270]

7. Halter-Hipsky, C.; Hue-Roye, K.; Coghlan, G.; Lomas-Francis, C.; Reid, ME. Two alleles with RHCE*nt818C > T change encode the low prevalence Rh antigen STEM Blood; 2009. p. 1226-7.

8. Hipsky, C Halter; Hue-Roye, K.; Lomas-Francis, C.; Reid, ME. RHD*DOL travels with $R H C E^{*} c e(818 C>T)$, which encodes a partial e, $\mathrm{hr}^{\mathrm{S}}-, \mathrm{hr}^{\mathrm{B}}+, \mathrm{STEM}+$ phenotype. Transfusion. 2010:48A.

9. Hipsky, C Halter; da Costa, DC.; Omoto, R.; Zanette, A.; Castilho, L.; Reid, ME. Prevalence of $R H D * D O L$ and $R H C E^{*} c e(818 T)$ in two populations. Immunohematology. 2011; 27:66-7. [PubMed: 22356522]

10. Noizat-Pirenne F, Lee K, Le Pennec P-Y, Simon P, Kazup P, Bachir D, Rousaud A-M, Roussel M, Juszczak G, Ménanteau C, Rouger P, Cartron J-P, Ansart-Pirenne H. Rare RHCE phenotypes in 
black individuals of Afro-Caribbean origin: Identification and transfusion safety. Blood. 2002; 100:4223-31. [PubMed: 12393640]

11. Hipsky, C Halter; Lomas-Francis, C.; Fuchisawa, A.; Reid, ME.; Moulds, M.; Christensen, J.; Nickle, P.; Vege, S.; Westhoff, C. RHCE*ceCF encodes partial c and partial e but not CELO an antigen antithetical to Crawford. Transfusion. 2011; 51:25-31. [PubMed: 20609196]

12. Hipsky, C Halter; Hue-Roye, K.; Lomas-Francis, C.; Huang, C-H.; Reid, ME. Molecular basis of the rare gene complex, $D I V(C)$-, which encodes four low prevalence antigens in the Rh blood group system. Vox Sanguinis. 2012; 102:167-70. [PubMed: 21729099]

13. Perco P, Shao CP, Mayr WR, Panzer S, Legler TJ. Testing for the D zygosity with three different methods revealed altered Rhesus boxes and a new weak D type. Transfusion. 2003; 43:335-9. [PubMed: 12675718]

14. Denomme G, Fernandes BJ. The assignment of RHD gene zygosity by densitometric quantification. Transfusion. 1999:51s.

15. Wagner FF, Moulds JM, Tounkara A, Kouriba B, Flegel WA. RHD allele distribution in Africans of Mali. BMC.Genet. 2003; 4:14. [PubMed: 14505497]

16. Matheson KA, Denomme GA. Novel 3' Rhesus box sequences confound RHD zygosity assignment. Transfusion. 2002; 42:645-50. [PubMed: 12084174]

17. Hipsky, C Halter; Velliquette, RW.; Larimore, K.; Olsen, C.; Lomas-Francis, C.; Reid, ME. Molecular background of RHCE in Bastiaan, the RH:-31,-34 index case, and two novel RHD alleles. Immunohematology. in press.

18. Peyrard T, Pham B, Juszczak G, Poupel S, Baudier A, Brochier G, Menanteau C, Rouger P, Le Pennec P. The "anti-e" and antibody to a high-prevalence Rh antigen made by $R H C E^{*} c e M O /$ $R H C E^{*} c E$ and $R H C E^{*} c e M O$ people are not anti-hr ${ }^{\mathrm{S}} / \mathrm{anti}^{\mathrm{h}} \mathrm{hr}^{\mathrm{B}}$ nor anti-Hr/anti-Hr${ }^{\mathrm{B}}$. Transfusion. 2010:144A.

19. Hue-Roye K, Reid ME, Westhoff CM, Lomas-Francis C. Red cells from the original JAL+ proband are also DAK+ and STEM+ Vox Sanguinis. 2011; 101:61-4. [PubMed: 21477150] 


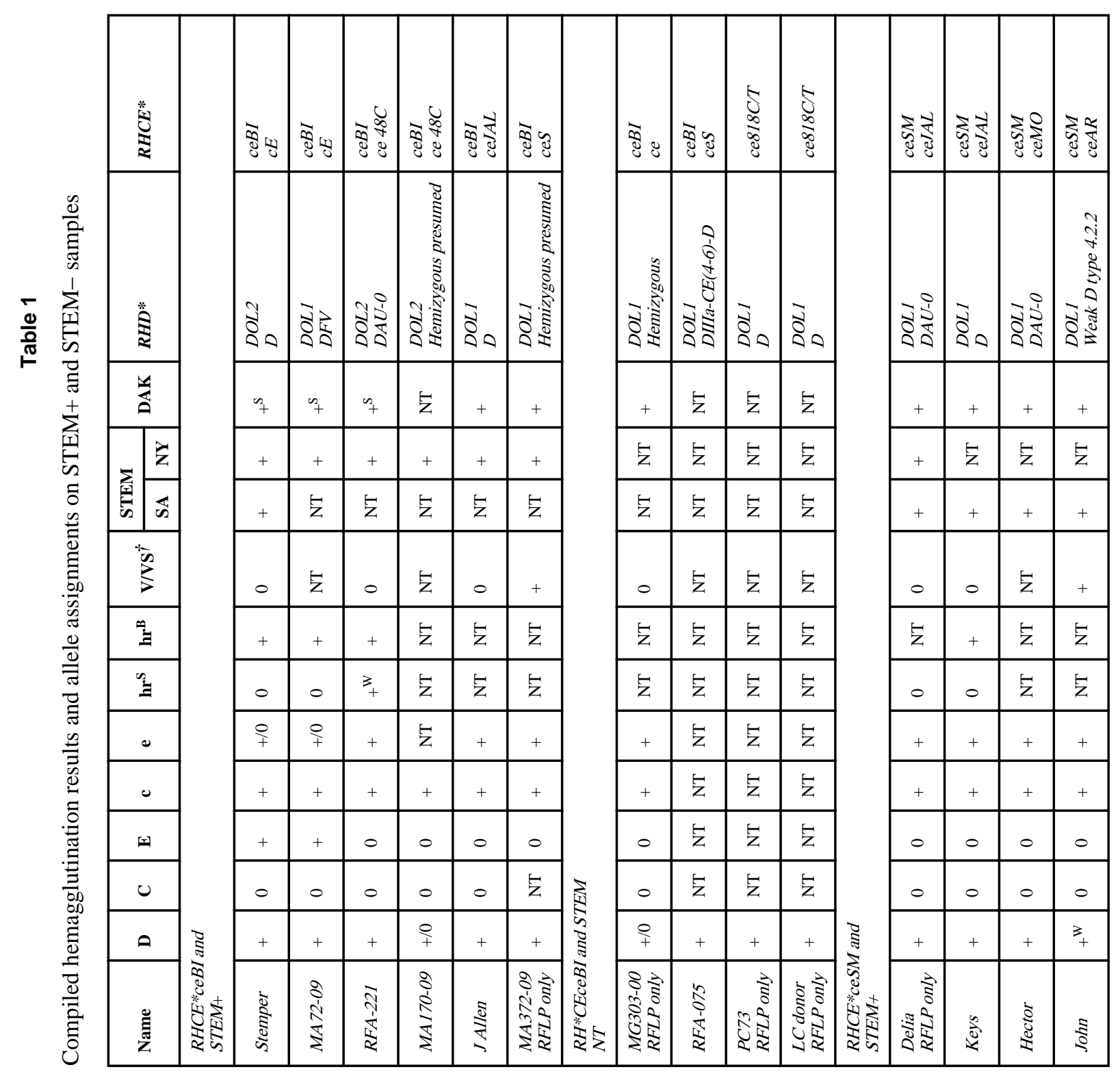




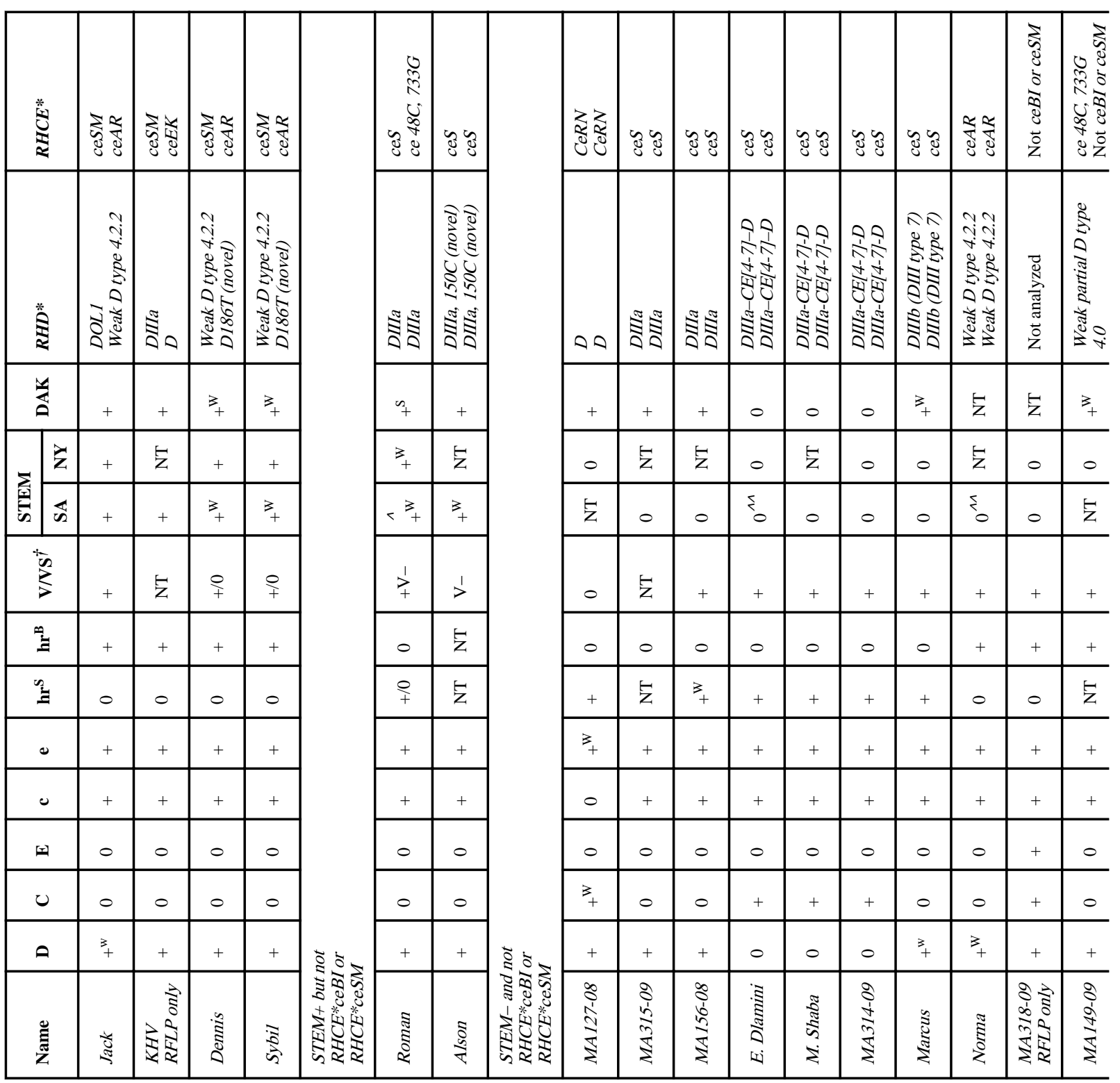




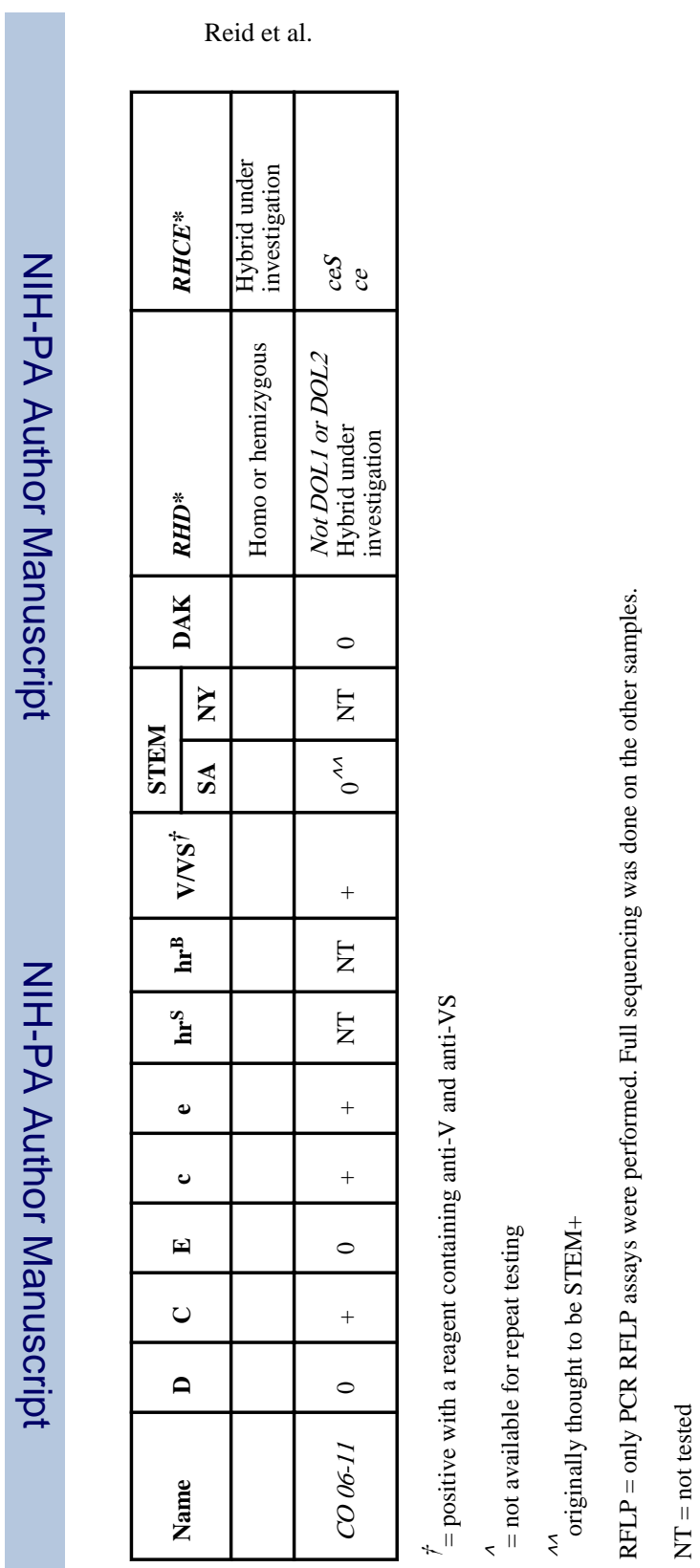

Page 10 


\section{Table 2}

Summary of STEM testing interpretations (no homozygous $R H C E^{*} c e 818 T$ or $R H D * 509 C, 667 G$ $\left[R H D^{*} D O L\right]$ were found) in a total of 31 samples

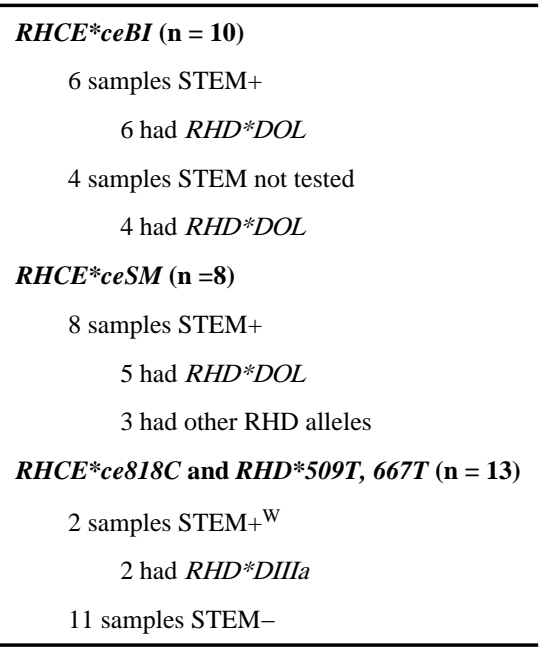

See Table 1 for alleles 